Research article

\title{
Endemic and epidemic dynamics of cholera: the role of the aquatic reservoir Cláudia Torres Codeço*
}

\author{
Address: Programa de Computação Científica Fundação Oswaldo Cruz, Rio de Janeiro, Brazil \\ E-mail: Cláudia Torres Codeço* - codeco@malaria.procc.fiocruz.br \\ *Corresponding author
}

Published: 2 February 2001

Received: I 3 July 2000

BMC Infectious Diseases 200I, I:I

Accepted: 2 February 200I

This article is available from: http://www.biomedcentral.com/I47/-2334/I/I

(c) 200 I Codeço, licensee BioMed Central Ltd.

\begin{abstract}
Background: In the last decades, attention to cholera epidemiology increased, as cholera epidemics became a worldwide health problem. Detailed investigation of $V$. cholerae interactions with its host and with other organisms in the environment suggests that cholera dynamics is much more complex than previously thought. Here, I formulate a mathematical model of cholera epidemiology that incorporates an environmental reservoir of $V$. cholerae. The objective is to explore the role of the aquatic reservoir on the persistence of endemic cholera as well as to define minimum conditions for the development of epidemic and endemic cholera.

Results: The reproduction rate of cholera in a community is defined by the product of social and environmental factors. The importance of the aquatic reservoir depends on the sanitary conditions of the community. Seasonal variations of contact rates force a cyclical pattern of cholera outbreaks, as observed in some cholera-endemic communities.

Conclusions: Further development on cholera modeling requires a better understanding of $V$. cholerae ecology and epidemiology. We need estimates of the prevalence of $V$. cholerae infection in endemic populations as well as a better description of the relationship between dose and virulence.
\end{abstract}

\section{Background}

Since Snow's seminal work (1855), when cholera epidemics were first associated with a contaminated water supply, this disease has been the focus of intense study. Cholera is a waterborne disease characterized by severe diarrhea. The etiological agent is $V$. cholerae $\mathrm{O} 1$ (more recently also $V$. cholerae O139), which colonizes the small intestine and produces an enterotoxin responsible for a watery diarrhea. Without prompt treatment, a person with cholera may die of dehydration in a matter of hours after infection. Cholera outbreaks are generally associated to contaminated food and water supplies. Appropriate sanitation and safe water are the main weapons against this disease.
Until the $19^{\text {th }}$ century, cholera was confined to the Indian sub-continent [1]. There, cholera outbreaks are seasonal with one or two peaks per year [2]. From this region, cholera has spread throughout the world seven times since 1817 [3]. The last pandemics began in 1961 in Indonesia, spread through the Asian continent during the 60's, reached Africa in 1970 [4] and Latin America in 1991. The occurrence of successive cholera outbreaks throughout Africa and Latin America during the 9o's raised the concern that cholera had established itself in these regions as an endemic disease.

Once cholera arrives into a new region, either carried by an infected person or by contaminated water and food, 
we may expect one of three possible outcomes: no outbreak, an outbreak possibly followed by few waves; or a cholera outbreak followed by subsequent outbreaks that may assume a persistent seasonal pattern. I call these, respectively, cholera-free; epidemic and endemic patterns. What demographic, environmental and sociological factors drive these outcomes? Studies suggest that the number of susceptibles; exposure to untreated water and sewage; and the presence of an aquatic reservoir of $V$. cholerae are important factors [1] [3] [5]. How important they are and how they interact with each other and with other variables to drive cholera epidemiology, are open questions.

Recently, it was found that toxigenic $V$. cholerae can survive in some aquatic environments for months to years, in association with zooplankton and other aquatic organisms [6]. Under stress, this pathogen assumes a viable but not culturable state, becoming undetectable to traditional bacteriological techniques. These findings suggest that the aquatic environment may be a reservoir of toxigenic $V$. cholerae in endemic regions. What is the role of the aquatic reservoir in promoting epidemic and endemic cholera? What regions are more likely to maintain endemic cholera? What are the best approaches to prevent and control cholera outbreaks? What are the best predictors of the fate of a community after the introduction of cholera?

Table I: Symbols used in the model

\begin{tabular}{|c|c|}
\hline Symbol & Description \\
\hline \multicolumn{2}{|c|}{ State Variables } \\
\hline $\mathrm{S}$ & number of susceptibles \\
\hline 1 & number of infected \\
\hline B & $\begin{array}{l}\text { concentration of toxigenic } \mathrm{V} \text {. cholerae in water } \\
(\text { cells } / \mathrm{ml})\end{array}$ \\
\hline \multicolumn{2}{|c|}{ Parameters } \\
\hline $\mathrm{H}$ & total human population \\
\hline $\mathrm{n}$ & Human birth and death rates (day-I) \\
\hline a & rate of exposure to contaminated water (day-I) \\
\hline $\mathrm{K}$ & $\begin{array}{l}\text { concentration of } \mathrm{V} \text {. cholerae in water that yields } 50 \% \\
\text { chance of catching cholera (cells } / \mathrm{ml})\end{array}$ \\
\hline$r$ & rate at which people recover from cholera (day-I) \\
\hline $\mathrm{nb}$ & $\begin{array}{l}\text { growth rate of } \mathrm{V} \text {. cholerae in the aquatic environ- } \\
\text { ment (day- } \mathrm{I})\end{array}$ \\
\hline $\mathrm{mb}$ & $\begin{array}{l}\text { loss rate of } \mathrm{V} \text {. cholerae in the aquatic environment } \\
\text { (day-I) }\end{array}$ \\
\hline \multirow[t]{2}{*}{ e } & $\begin{array}{l}\text { contribution of each infected person to the popula- } \\
\text { tion of } V \text {. cholerae }\end{array}$ \\
\hline & in the aquatic environment (cell/ml day-I person-I) \\
\hline
\end{tabular}

Here, I start to address these questions by proposing a mathematical model that incorporates what are considered important determinants of cholera dynamics. Despite the great effort towards understanding cholera dynamics, the mechanisms driving $V$. cholerae dynamics in water is still poorly known. Thus, it is appropriate to start a modeling exercise with a simple model (with few variables) and then add complexity as needed. Following this methodology, I started with a basic Susceptible-Infective-Recovered model coupled to an aquatic population of $V$. cholerae. The objective is to explore the role of the aquatic reservoir on the persistence of endemic cholera as well as to define minimum conditions for the development of epidemic and endemic cholera. Mathematical models are powerful tools for the development of epidemiological theories [7]. They can synthesize the current empirical knowledge about the disease into a coherent mechanistic framework. These models may help us to infer causal relationships and to suggest experimental designs to test alternative hypotheses.

This work is structured in three parts. First, the mathematical model for epidemic and endemic cholera is presented. Secondly, the proposed model is applied to three hypothetical communities to simulate cholera-free, epidemic and endemic situations. Thirdly, I consider possible causes of endemic oscillations. I finish this work discussing insights brought from model analyses into cholera dynamics.

\section{Mathematical Model}

The model proposed here is an extension of Capasso's model [8], used to describe the 1973's cholera epidemics in Italy. In Capasso's version, two equations describe the dynamics of infected people in the community and the dynamics of the aquatic population of pathogenic bacteria. In our formulation, the dynamics of the susceptible population is included since I wish to study long term dynamics [7]. The mathematical model is:

$$
\begin{aligned}
& \frac{d S}{d t}=n(H-S)-a \lambda(B) S \\
& \frac{d I}{d t}=a \lambda(B) S-r I \\
& \frac{d B}{d t}=B(n b-m b)+e I
\end{aligned}
$$

$$
S(0)=H, I(0)>0, B(0)=0
$$

Symbols are listed in Table 1 and a diagrammatic representation of the model is shown in Figure 1. Equation 1a describes the dynamics of susceptibles in a community of 


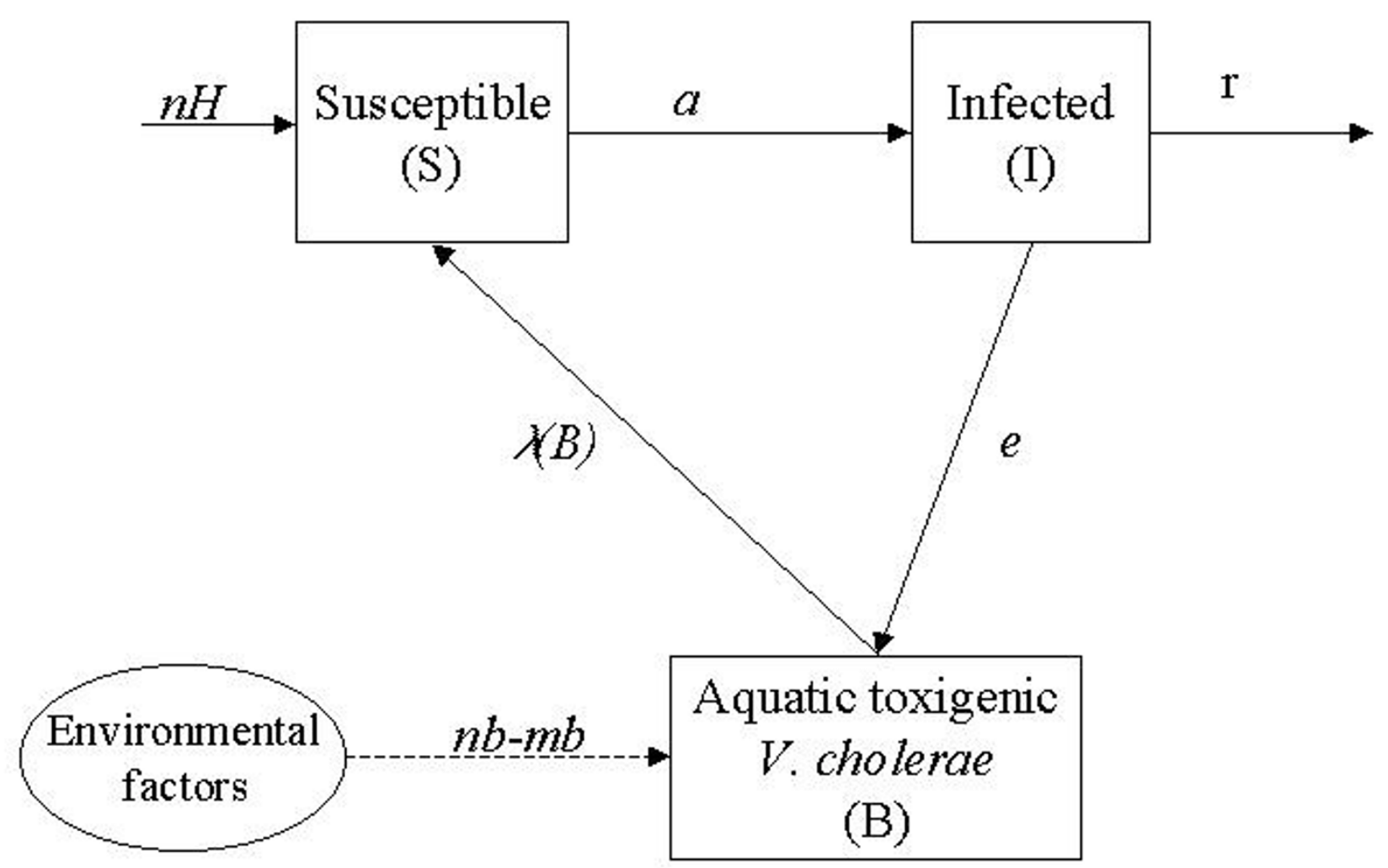

Figure I

Model diagram (symbols are listed in table I). All individuals in population $H$ are born susceptible. Susceptible people (S) become infected as they are exposed to contaminated water (i.e., to $B$ ). Infected people recover at a rate $r$. Recovered population is not explicitly included but its size can be estimated by $\mathrm{H}-\mathrm{I}$-S (since total population is constant). While infected, individuals contribute to the enhancement of bacterial population through excretion. Bacterial population in the aquatic reservoir (B) may also grow in the water at a rate determined by environmental factors (temperature, for example).

constant size $H$. Susceptible individuals are renewed at a rate $n$. Renewal may occur as result of birth, immigration and/or loss of acquired immunity (cholera apparently does not confer life-long immunity). Susceptible people becomes infected at a rate $a \lambda(\mathrm{B})$, where $a$ is the rate of contact with untreated water and $\lambda(B)$ is the probability of such person to catch cholera. Probability of catching cholera depends on the concentration of $V$. cholerae in the consumed water. Experimental studies suggest that it is necessary a heavy inoculum of $V$. cholerae in order to develop cholera [9]. Here, this dependence is represented by a logistic dose response curve (Figure 2)

$$
\lambda(B)=\frac{B}{K+B}
$$

where $K$ is the concentration of $V$. cholerae in water that yields $50 \%$ chance of catching cholera. I assume that the only route for infection is the ingestion of contaminated water from non-treated sources.

Equation $1 \mathrm{~b}$ describes the dynamics of infected people in the community. This category includes not only cholera cases but also those with asymptomatic and mild infections. In reality, only 1 to $30 \%$ of $V$. cholerae infections actually develops into severe cholera cases [10]. By combining all infection types into a single model compartment, I am assuming that they follow the same dynamics, i.e., that the case-to-infection ratio remains constant through the epidemics. This is reasonable if infection virulence is strongly determined by host factors (sensitivity to cholera toxin, blood type, etc) and bacterial factors (biotype, etc), that are not expected to change during the period of interest [11] [12] [13]. A potential problem with this assumption is that inoculum size may affect the case-to-infection ratio [14]. It is not clear, however, if inoculum size is directly related to virulence or if it just increases the chance of intestine colonization. 


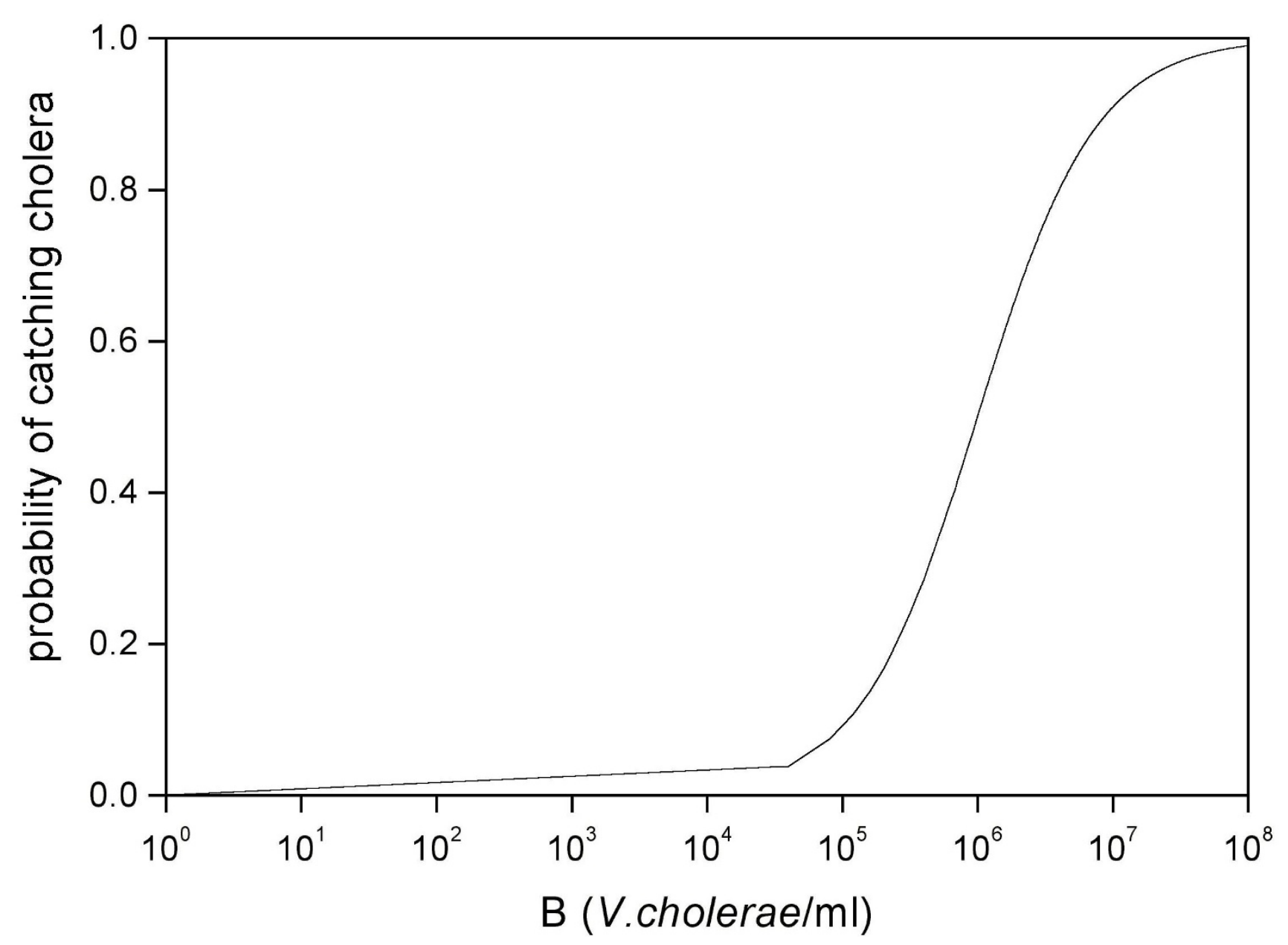

Figure 2

Dose-dependent infection rate.

Here, I assumed that inoculum size affects the per capita infection rate (i.e., the probability of colonization), but not the severity of symptoms. Equation $1 \mathrm{~b}$ states that the infected population increases as susceptibles become infected (first term in the equation) and decreases as they recover from the disease or die.

The third equation describes the dynamics of pathogenic $V$. cholerae in the aquatic reservoir, in this case, the set of untreated waters consumed by the population. Environmental $V$. cholerae is found in ponds, wells, rivers, estuaries and coast waters. These environments have quite distinct physical, chemical and biological characteristics and $V$. cholerae dynamics is probably controlled by different factors in each of them. Classical studies on the survival of culturable $V$. cholerae in aquatic environments suggest that $V$. cholerae cannot maintain a stable population in the environment. Observed rates of population extinction in microcosms are variable, ranging from $0.02 \mathrm{day}^{-1}$ to $>3 \mathrm{day}^{-1}$ [15]. Islam [16], however, showed that population decay does not necessarily imply death, but also the transition towards a non-culturable state. Using fluorescent antibody techniques, he found $V$. cholerae surviving for more than 15 months within the mucilagenous sheaths of a filamentous alga (extinction rate $<0.002$ ). Here, equation 1c states that bacterial density in the water results from the balance between local birth and death processes and the inflow of contaminated sewage. The parameter $e$ defines the average contribution of each infected person to the aquatic population of $V$. cholerae.

At last, equations (2) specify the initial conditions (all individuals are initially susceptible).

\section{Model predictions}

The model predicts three qualitative outcomes after the introduction of few cholera infectives into a susceptible population: cholera does not spread; epidemic cholera and endemic cholera. Here I describe each dynamics separately and illustrate them using three hypothetical communities, whose parameters are shown in Table 2. 
Table 2: Parameters for three hypothetical communities.

\begin{tabular}{cccc}
\hline Parameters & Community I & Community 2 & Community 3 \\
\hline $\begin{array}{c}\text { Expected cholera } \\
\text { dynamics }\end{array}$ & cholera-free & epidemic & endemic \\
H & 10,000 & 10,000 & 10,000 \\
$\mathrm{n}$ & $0.000 \mathrm{I}$ & $0.000 \mathrm{I}$ & 0.001 \\
$\mathrm{a}$ & 0.5 & $\mathrm{I}$ & $\mathrm{I}$ \\
$\mathrm{K}$ & $\mathrm{I} \times 106$ & $\mathrm{I} \times 106$ & $1 \times 106$ \\
$\mathrm{r}$ & 0.2 & 0.2 & 0.2 \\
$\mathrm{nb}-\mathrm{mb}$ & -0.33 & -0.33 & -0.33 \\
$\mathrm{e}$ & 10 & 10 & 10 \\
$\mathrm{SC}$ & 13200 & 6600 & 6600 \\
\hline
\end{tabular}

\section{First case: cholera-free population}

Consider a community that does not experience cholera for generations. All individuals are susceptibles. There are neither infective or immune individuals nor toxigenic bacteria in the water.

$S^{*}=H ; I^{*}=0 ; B^{*}=0$

Superscript ${ }^{*}$ indicates equilibrium quantities. The first question of interest is: What will happen to this community if a small number of infectives comes in? Stability analysis of equation system 1 (see 1additional material: Appendix) indicates that if the number of susceptibles in this population is greater than a critical number $S_{C}$, an outbreak will occur. Otherwise, the number of cases will decrease and return to zero (Figure 3). This threshold is given by:

$$
S c=\frac{r K(m b-n b)}{e}
$$

The $S_{\mathrm{c}}$ threshold increases proportionally to the barriers to cholera infection $(K)$, and recovery $(r)$ as well as the net mortality rate of $V$. cholerae in the water. It decreases, on the other hand, as contamination of water supplies as well as contact with these waters increase. In other words, the better water quality and sewage treatment are, the greater must be the pool of susceptibles in order to trigger a cholera outbreak.

Rearranging equation 5 , we find the maximum degree of contamination each infected person may cause to the water reservoir without causing public health hazards: $e=\frac{r K(m b-n b)}{a S_{C}}$

Asymptomatic cases of $V$. cholerae infection yield $10^{2}$ $10^{5} \mathrm{~V}$. cholerae per gram of feces [15]. In severe cases, yield increases to $10^{6}-10^{9}$ cells $/ \mathrm{ml}$ of rice-water stool [15]. If we know the proportion of infections that are asymptomatic, mild and severe, we could obtain a rough estimation of $e$ as

$e=\frac{e_{\text {asymptomatic }} I_{\text {asymptomatic }}+e_{\text {mild }} I_{\text {mild }}+e_{\text {severe }} I_{\text {severe }}}{I_{\text {asymptomatic }}+I_{\text {mild }}+I_{\text {severe }}}$

Consider a hypothetical community with 10,000 members sharing the same water source. Suppose that the water reservoir is directly contaminated with sewage. If $a=$ 1 , cholera outbreak in this community will develop only if individual contribution to water contamination exceeds ca. 7 cells $/ \mathrm{ml} \mathrm{day}^{-1}$. Now, consider that each infected person yields $1 \times 10^{4}$ cells person ${ }^{-1}$ day $^{-1}$. This water will remain infective unless it is diluted to a value less than 7 cells $/ \mathrm{ml}$. Such dilution requires $4 \times 10^{4 / 7}=$ ca. 5700 liters of water per infected person per day.

A real pond, however, is not perfectly mixed as this model assumes. If water used for consumption is taken from a point close to the excretion site, the risk of acquiring the infection will be higher even if the pond is large. Moreover, $V$. cholerae is found associated to phytoplankton, macrophyte, zooplankton, crustacea and other aquatic organisms. On the surface of these organisms, density of bacteria may be 100 to 1000 times greater than in the aquatic medium. These organisms are not evenly distributed within the water body. Phytoplankton tends to concentrate on the water surface, zooplankton migrates along the water column on a daily basis. If water is taken from phyto- or zooplankton-rich patches, risk of catching cholera will increase, enhancing the probability of triggering an outbreak in the community.

This model is too simple to provide quantitative predictions on cholera dynamics. Nevertheless, its qualitative results verifies known alternative approaches to the prevention of cholera outbreaks:

1. Minimize water contamination as well as consumption of untreated waters: This is the classical approach to cholera control. Good sanitation reduces the parameter $e$ and water treatment reduces parameter $a$. The smaller these parameters are, the larger must be the susceptible pool in order to a cholera outbreak to develop (equation $5)$. 


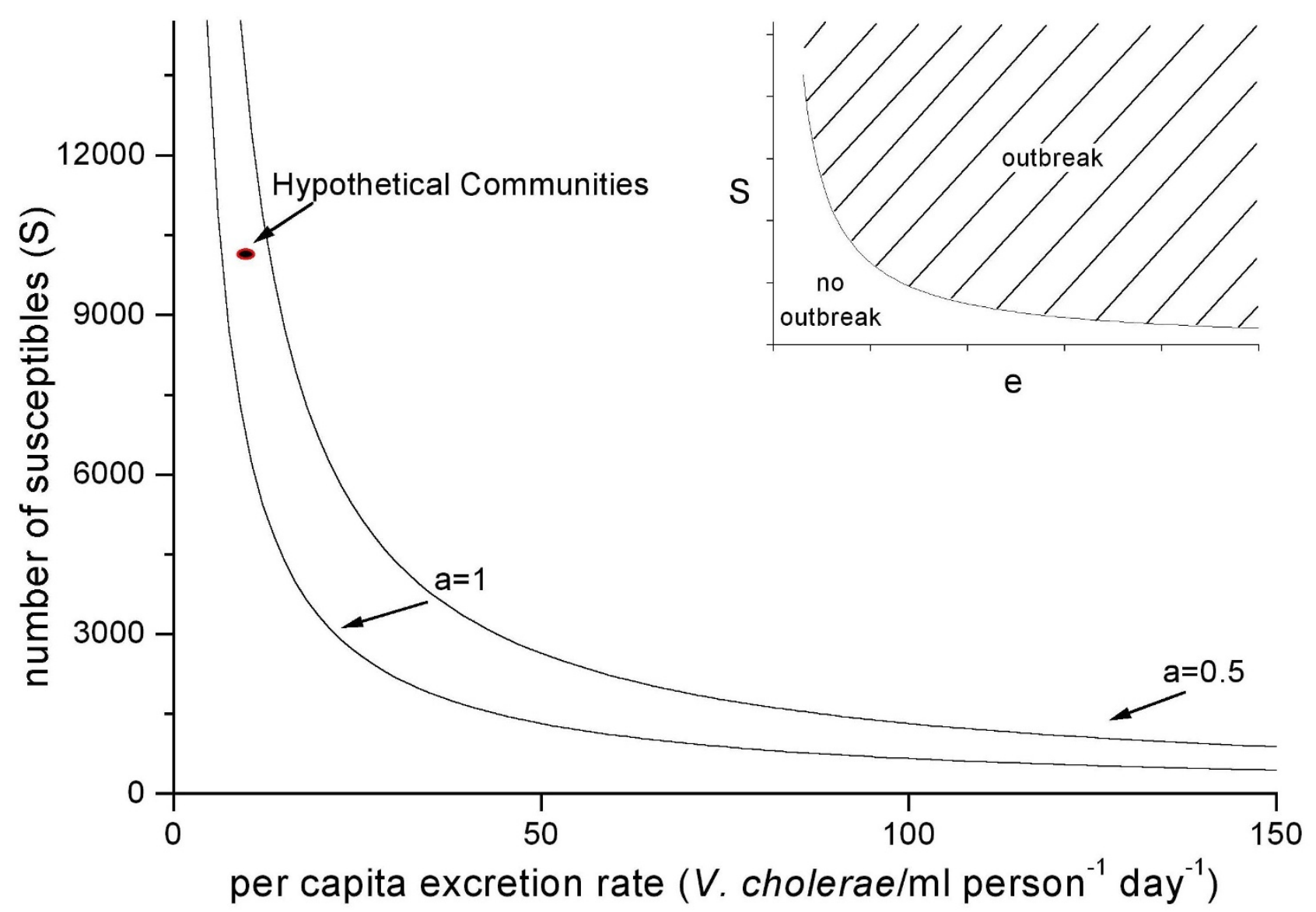

Figure 3

Effect of e (mean contribution of each individual to water contamination) on the threshold population size $S_{\mathrm{C}}$ (equation 5 ). Curve $a=0.5$ uses parameters from community I; curve $a=1$ uses parameters from communities 2 and 3 (table 2). Each curve defines values of $e$ and $S_{C}$ at which $R_{0}=I$ (see ladditional material: Appendix). A community located above its curve will suffer an outbreak following the introduction of infectives. A community located below its curve, will not. The three hypothetical communities occupy the same position in this graph as they share the same initial susceptible pool size and degree of water contamination. However, community I does not suffer a cholera outbreak because the lower contact with contaminated water $(a=0.5)$ shifts its threshold curve outwards. Communities 2 and 3 are located above their threshold curve $(a=1)$; the introduction of infectives will trigger an outbreak.

2. Dilute cholera diarrhea with large amounts of water to make water uninfective.

\section{Second case: Epidemic cholera}

Now, consider a community whose susceptible pool exceeds the threshold $S_{\mathrm{C}}$. The introduction of infectives into this community will trigger a cholera outbreak. Figure 4 shows the dynamics of infectives, susceptibles and bacteria in community 2 (Table 2) after the introduction of a few infectives. Cholera outbreak follows a traditional epidemiological curve. The initial reproduction rate of the disease is positively affected by the degree of contamination of the water supply $(e)$ as well as the frequency of contact with these waters $(a)$ :

$$
R_{0}=\frac{a e}{r K(m b-n b)} S_{0}
$$

Together with the outbreak curve, we observe a bacterial bloom in the water. Since growth rate is negative, bacterial population will eventually decline when the susceptible pool decreases below the critical size $S_{\mathrm{C}}$ (Figure $4 \mathrm{~b}$ ). From this point on, bacterial extinction rate exceeds human excretion and the environmental $V$. cholerae population eventually goes extinct. Community 2, then, returns to the cholera-free steady state. 


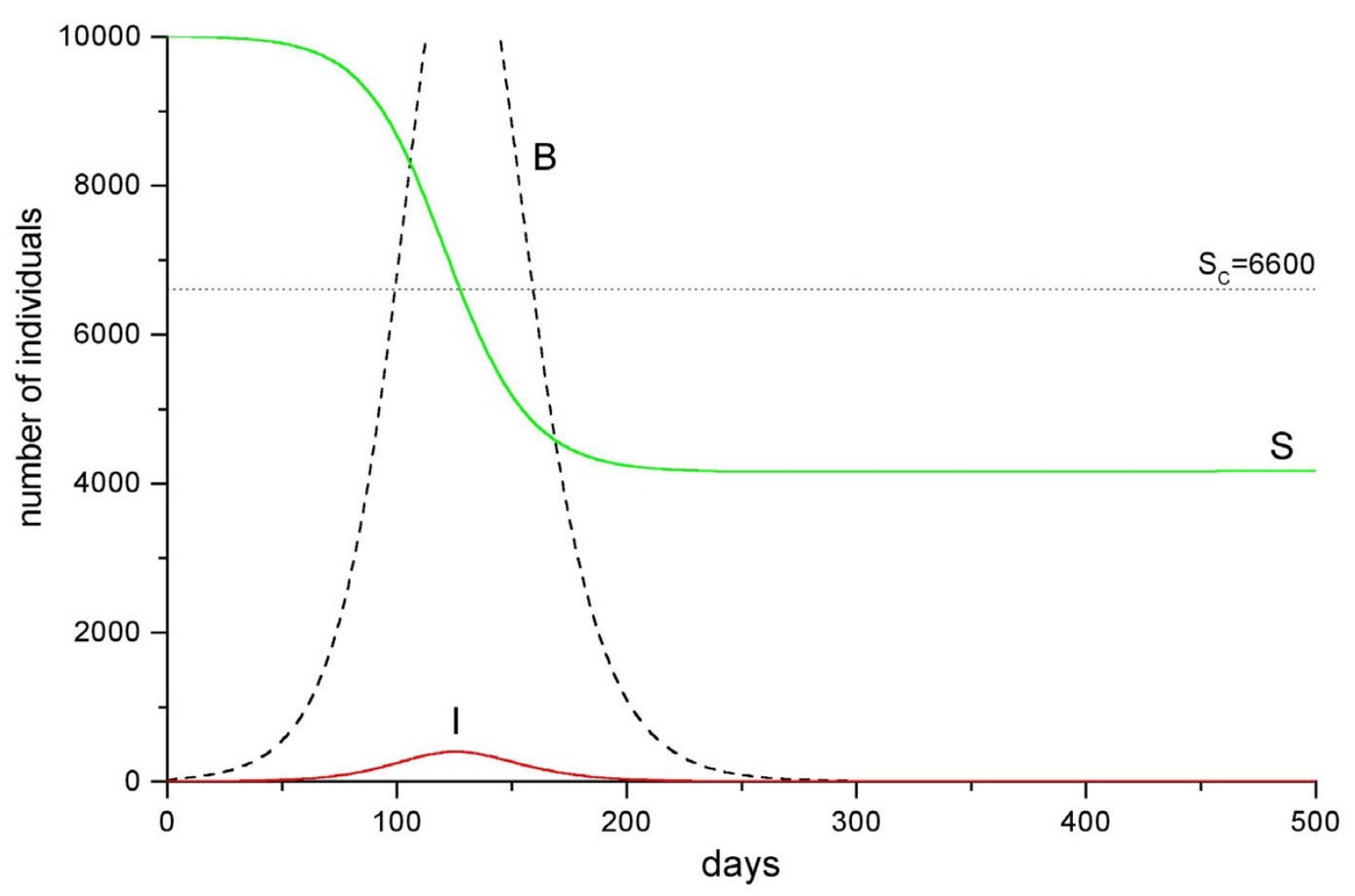

\section{Figure 4}

Simulation of a cholera outbreak in Community 2 (numerical solution of equation system I with parameters from table 2). The simulation starts with 10,000 susceptibles. The arrival of an infected individual triggers an outbreak. Bacterial density in the water (dashed black line) increases as result of human excretion. The epidemic curve (red solid line) starts to decline when the number of susceptibles crosses down the threshold line Sc.

\section{Third case: Endemic cholera}

Consider our hypothetical community 3 (Table 2). Its parameters are identical to those set for community 2, except that $n$ is greater. Since $S_{\mathrm{o}}>S_{\mathrm{C}}$, the introduction of infectives in community 3 starts a cholera outbreak. In this case, however, cholera does not vanish after the first peak. It actually returns in successive waves and eventually converges to a positive endemic equilibrium (Figure 5). Setting the derivatives of equation system 1 to zero and solving it algebraically, we obtain the endemic equilibrium:

$$
\begin{aligned}
S^{*} & =H-\frac{r}{n} I^{*} \\
I^{*} & =\frac{n(r K m b-r K n b+a e H)}{e(n+a) r}=\phi\left(H-S_{C}\right) \\
B^{*} & =\frac{e I^{*}}{m b-n b}
\end{aligned}
$$

The equilibrium number of infected individuals in the population is a fraction $\phi$ of the surplus population $(\mathrm{H}-\mathrm{S}$ $\mathrm{C})$, where $\phi$ is

$$
\Phi=\frac{n a}{n+a r}
$$

Fraction $\phi$ increases as the susceptible turnover rate $n$ increases and tends to $a$ at large values of $n$. This fraction is a function of human parameters, only.

Although in theory any community with $S_{\mathrm{O}}>S_{\mathrm{C}}$ and $n$ $>\mathrm{O}$ is expected to become endemic, this is not likely to occur in practice. To maintain an endemic state, $I^{*}$ must be much greater than 1 , otherwise infection will fade-out due to stochastic processes [7]. 


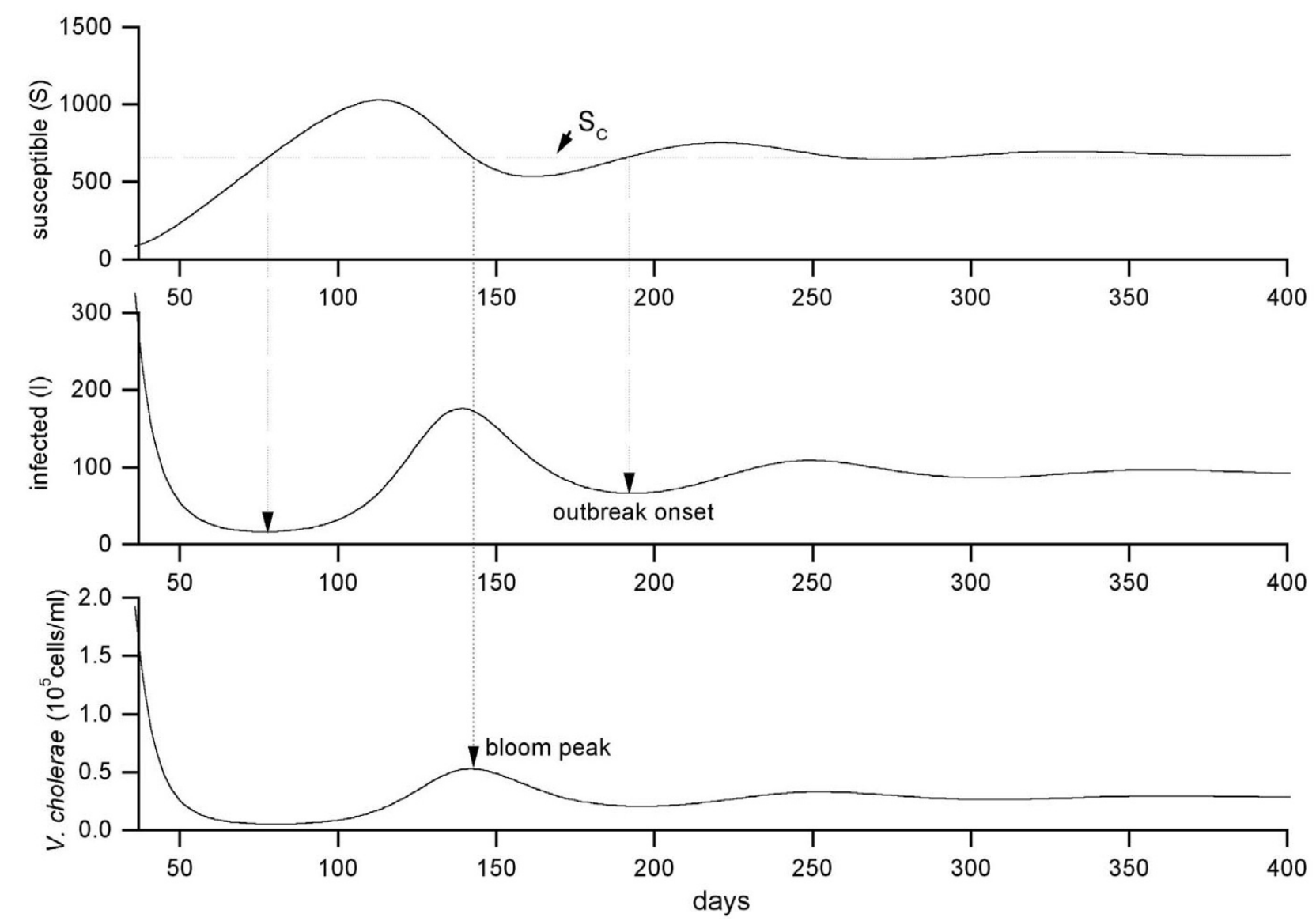

Figure 5

Simulation of cholera dynamics in the hypothetical community 3, using parameters from table 2 . The fast turnover of susceptibles allows cholera to persist. After the initial outbreak (not shown, but similar to figure 4), cholera prevalence oscillates until it reaches a steady-state. Oscillations are triggered when the number of susceptibles exceed the Sc threshold (dashed line).

Stability analysis (see 1additional material: Appendix) indicate that the endemic equilibrium is stable to small perturbations if $S_{\mathrm{C}}>S_{\mathrm{O}}$ and $m b>n b$. The route to the endemic equilibrium involves dumped oscillations (Figure 5). Cholera waves arise when the susceptible population grows above the threshold $S_{\mathrm{C}}$, triggering a new outbreak. Eventually, the number of infectives stabilizes into a fixed fraction of the population. Bacterial dynamics in the water is also oscillatory and follows the human excretion pattern.

In summary, our model predicts that cholera outbreaks should occur in communities whose susceptible pool is greater than the susceptible threshold $\left(S_{\mathrm{C}}\right)$. The magnitude of the threshold depends on a combination of environmental, sociological and strain-specific factors. The environmental reservoir reduces the susceptible threshold. In the presence of a permanent reservoir of $V$. cholerae ( $n b$ tending to $m b$ ), the threshold tend to zero, and community of any size in contact with the reservoir should be subject to an outbreak.

\section{Environmental triggers of cholera outbreaks}

In endemic regions, cholera outbreaks are often associated to climatic events [17]. In some parts of Africa, outbreaks occur during the dry season or right after heavy rainfalls [4][18]. In the Americas, disasters caused by the El Niño preceded large cholera outbreaks [19]. In Bangladesh, cholera season coincides with the post-monsoon period [3]. Flooding and drought are likely to affect cholera dynamics in a complex way. Flooding of streets and cities washes contaminated feces and sewage into the rivers. It may also disrupt water distribution service and aggravate hygiene conditions [18]. Drought, on the other hand, reduces the availability of potable water; aggravates hygiene conditions; increases the number of people sharing the same water supply; and may increase per capita water contamination. All these factors contribute to a greater rate of contact with unsafe waters (parameter $a$ ) and increased (floods) or reduced (floods or droughts) dilution of the per capita bacterial yield (parameter $e$ ). 
Besides weather changes, seasonal variations of water temperature has been also associated to cholera outbreaks. Cholera outbreaks in Peru [20] and Bangladesh [21] are preceded by periods of warm waters, during which toxigenic $V$. cholerae is detected in the environment. Colwell [21] explains this pattern by an association between zooplankton seasonal growth (that generally peaks during the warm season) and $V$. cholerae dynamics.

Floods, droughts, temperature and biotic interactions have potentially quite different impacts on cholera seasonality. To untangle the potential roles of these factors, I conducted a series of simulations of equation system 1 where the following parameters are assumed to vary seasonally: 1) the contact rate with contaminated water (simulating seasonal variations in water quality); 2) the per capita excretion rate (simulating seasonal variations in per capita water contamination); 3) the growth rate of $V$. cholerae (simulating seasonal variations in autochtonous $V$. cholerae abundance in the water). In all simulations, a sine function with period equal to 365 days was used to simulate the seasonal oscillations.

\section{Scenario I. Seasonal oscillation of the contact rate (a)}

Here, I substituted the parameter $a$ in equation 1 by the sin function:

$a(t)=0.5+0.5 \sin (2 \pi t / 365)$

The forced equation system was numerically solved (using the software ModelMaker) using parameters for the hypothetical community 3 (Table 2). To test the sensitivity of the obtained results to parameter change, I also carried out a sensitivity analysis where the model was simulated 42 times, keeping all parameters at their default values (Table 2) and changing only one (Table 3).

Table 3: Parameter values used in the sensitivity analysis.

Parameters

$\begin{array}{cc} & \\ \mathrm{H} & \mathrm{I}, 000 ; 20,000 ; 40,000 ; 60,000 ; 80,000 ; 100,000 \\ \mathrm{n} & 0.000 \mathrm{I} 0.002 ; 0.004 ; 0.006 ; 0.008 ; 0.0 \mathrm{I} \\ \mathrm{a} & 0.1 ; 0.2 ; 0.4 ; .0 .6 ; 0.8 ; \mathrm{I} \\ \mathrm{K} & \mathrm{I}, 000 ; 20,000 ; 40,000 ; 60,000 ; 80,000 ; 100,000 \\ \mathrm{r} & 0.02 ; 0.05 ; 0.09 ; 0.13 ; 0.16 ; 0.2 \\ \mathrm{nb}-\mathrm{mb} & -0.02 ;-0.13 ;-0.25 ;-0.37 ;-0.48 ;-0.6 \\ \mathrm{e} & \mathrm{I} ; 20 ; 40 ; 60 ; 80 ; 100\end{array}$

All 42 simulations resulted in periodic dynamics with an annual peak of cholera (Figure 6). In general, peaks tended to occur earlier in larger populations with high water contamination. Low values of $\mathrm{K}$ and long bacterial residence time also contributed to the early onset of cholera outbreak. In most simulations (except those with the minimal values of $e, H$, and $n$ ), cholera outbreak was followed by a period of variable length during which infection incidence remained ca. constant. This "plateau" eventually vanished and a period with no human infection was noted until the next annual outbreak. Figure 6 shows the effect of population size on the infection dynamics. The greater the population size $(H)$ or the susceptible turnover rate $(n)$, the shorter is the period without any infection.

\section{Scenario 2. Seasonal oscillation of the per capita contam- ination rate (e)}

In this scenario, the parameter $e$ in equation system 1 was replaced by the periodic function:

$e(t)=e+e \sin (2 \pi t / 365)$

Simulations were performed using the same parameter values listed for the first scenario (now $a$ is constant). Seasonal variation of $e$ forces an annual outbreak whose peak occurs from 3 months earlier up to one month later the $e(t)$ peak. The greater the population size and the susceptible turnover rate are, the earlier the outbreak starts. Low $a$ and low $e$ are the main factors delaying the cholera onset. A period with no infections precedes each annual big outbreak (Figure 7).

A prevalence "plateau" often follows the annual cholera outbreak. The dynamics is very similar to that observed for the scenario 1. For some parameter combinations, however, this plateau rises to form a second outbreak of minor intensity. Conditions favoring the occurrence of this second peak include high $\mathrm{K}$, high extinction rate and fast recover rate.

\section{Scenario 3. Seasonal oscillation of V. cholerae extinction rate (nb-mb)}

In this last scenario, the term netnb $=(n b-m b)$ in equation system 1 was replaced by the periodic function:

$n e t n b(t)=n e t n b+n e t n b \sin (2 \pi t / 365)$

The goal is to simulate, in a phenomenological way, the seasonal variation of $V$. cholerae abundance in the environment due to autochtonous growth. Seasonal growth of $V$. cholerae in the environment has been linked to factors as temperature, copepod abundance and chloro- 


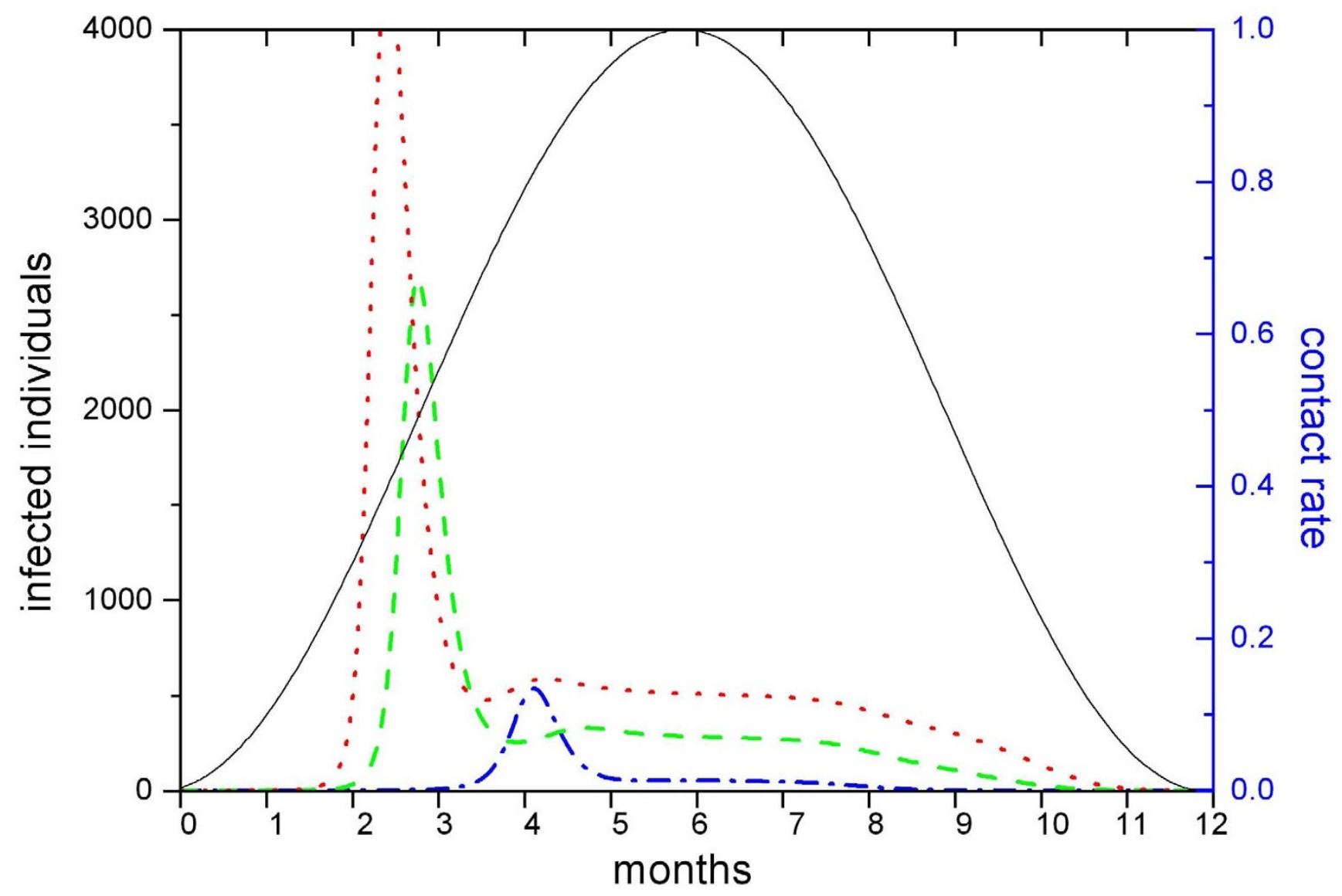

Figure 6

Simulation of a community that experiences seasonal contact with contaminated waters (due to periodic flooding, for example). Periodic fluctuation of the contact rate causes oscillations in the number of infections. During the low contact period, no infections occur. As contact increases (due to rising waters in a flooding area, for example), the probability of catching cholera increases. Outbreaks tend to occur sooner in larger populations. Dot-dashed line shows the number of infections in a population with I,000 individuals, dashed line in a 5,500 population and dotted line in a 100,000 population. In large populations, the seasonal outbreak may be followed by a period with relatively constant incidence as contact rate continues high.

phyll. However, the mechanistic relationships between these variables are still not clearly defined and I opted for not including them explicitly in the model.

Forty-two simulations were again performed. All simulations showed annual outbreaks of $V$. cholerae infection 2 to 4 months after the onset of $V$. cholerae growth in the water. Small populations, and low rate of contact with contaminated water helped to delay cholera outbreak. Differently from the previous two scenarios, the number of infected people never dropped to zero in large populations (Figure 8).

\section{Discussion}

In the last decades, attention to cholera epidemiology increased, as cholera epidemics became a worldwide health problem. Detailed investigation of $V$. cholerae interactions with its host and with other organisms in the environment suggests that cholera dynamics is much more complex than it was previously thought. We know now that cholera toxin is codified by a lisogenic phage that can be transferred horizontally between bacteria [22][23]. We also know that $V$. cholerae can survive in the water for a long time, assuming a non-culturable form, living in association with aquatic organisms. These findings increase the complexity of the cholera system, that passes from a two-factor system (human - pathogen) to a three or four factor system (virus-bacteriaaquatic organisms-humans?). Despite the great effort towards understanding cholera dynamics, we are still scratching the surface of this problem. Most studies associating cholera dynamics to environmental events are based on correlations. The causal mechanisms behind these correlations are still poorly understood. Unfortunately, many experimental studies performed in the past, on the tolerance and survival of $V$. cholerae in the 


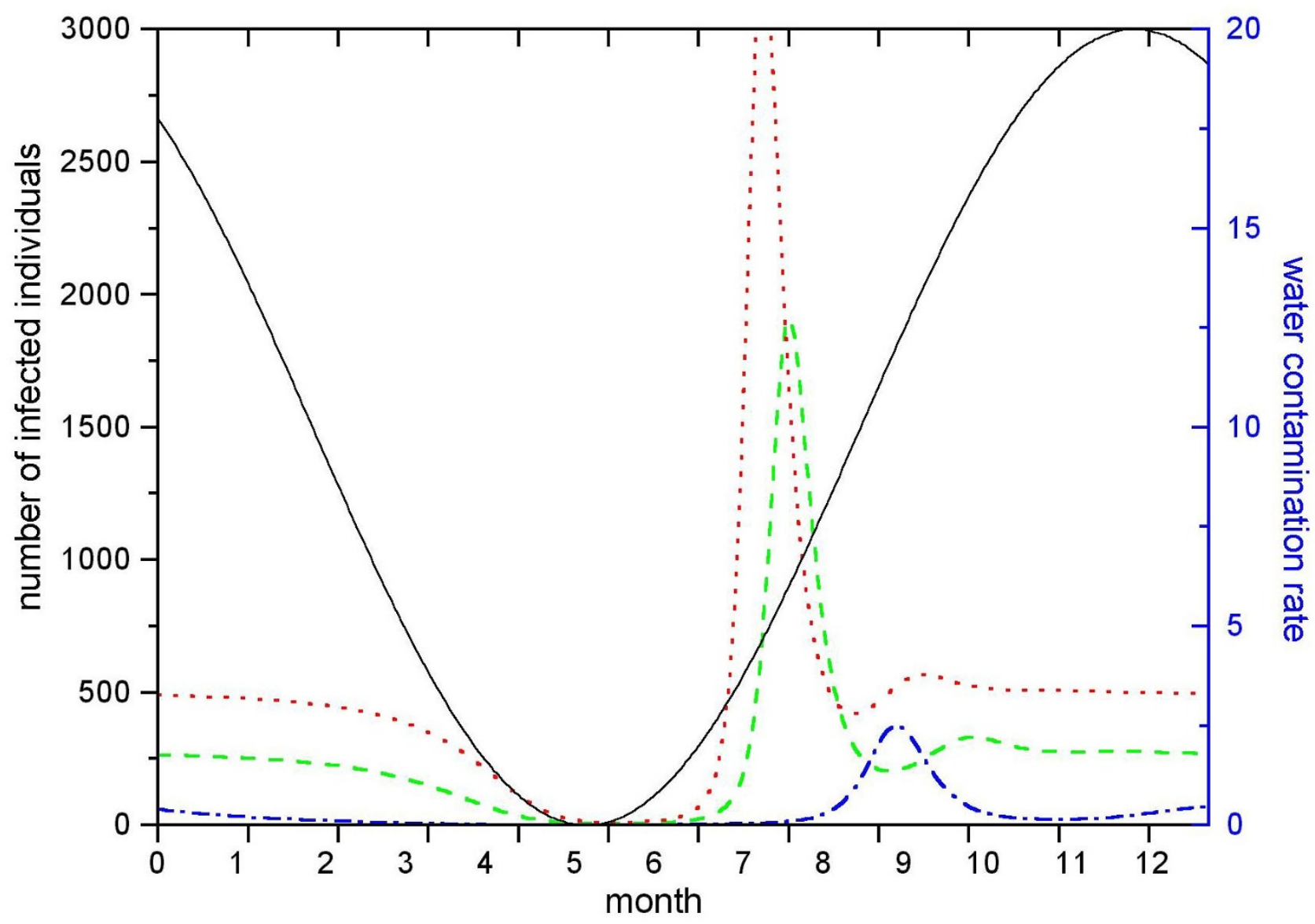

Figure 7

Dynamics of infection in a hypothetical population with 1,$000 ; 5,500$ and 100,000 individuals under seasonal variation of the water contamination rate (due to water shortage, for example). Symbols are the same as figure 6 . Seasonal decay of water quality triggers periodic outbreaks that are followed by a period of approximately constant prevalence.

environment (important for the establishment of causal relationships), may be misleading because they used bacteriological techniques for detecting $V$. cholerae. These findings may require further considerations on the light of the existence of unculturable forms of $V$. cholerae.

Experimental effort towards understanding cholera epidemiology has been accompanied by a theoretical effort. Authors have proposed new conceptual models of cholera epidemiology where the aquatic environment has an important role on the emergence of new strains of toxigenic $V$. cholerae [3] and on the seasonality of cholera outbreaks [5] [6].

The model introduced here is based on a previous cholera model [8], modified to incorporate an environmental reservoir. We still know very little about the dynamics of $V$. cholerae in the environment. Consequently, I opted for modeling the population dynamics of $V$. cholerae in the water in the simplest way possible, i.e., with density independent growth and death rates. This model is quite abstract but provides some insights about the role of an environmental reservoir on cholera epidemiology.

According to the model, a permanent environmental reservoir of toxigenic $V$. cholerae $(m b=n b)$ should increase $\mathrm{R}_{\mathrm{O}}$ towards infinity (equation 8 ). It means that any susceptible human population exposed to this water would be prone to cholera outbreaks. The magnitude of the problem, in terms of public health, would vary according to the probability of secondary transmission. In a community with good sanitation, cholera would show up as occasional primary cases without secondary transmission. In poor communities, on the other hand, endemism would result from the close contact between susceptibles and the source of contamination. An infinite $R_{O}$ is an overestimation of this simplistic deterministic model. 


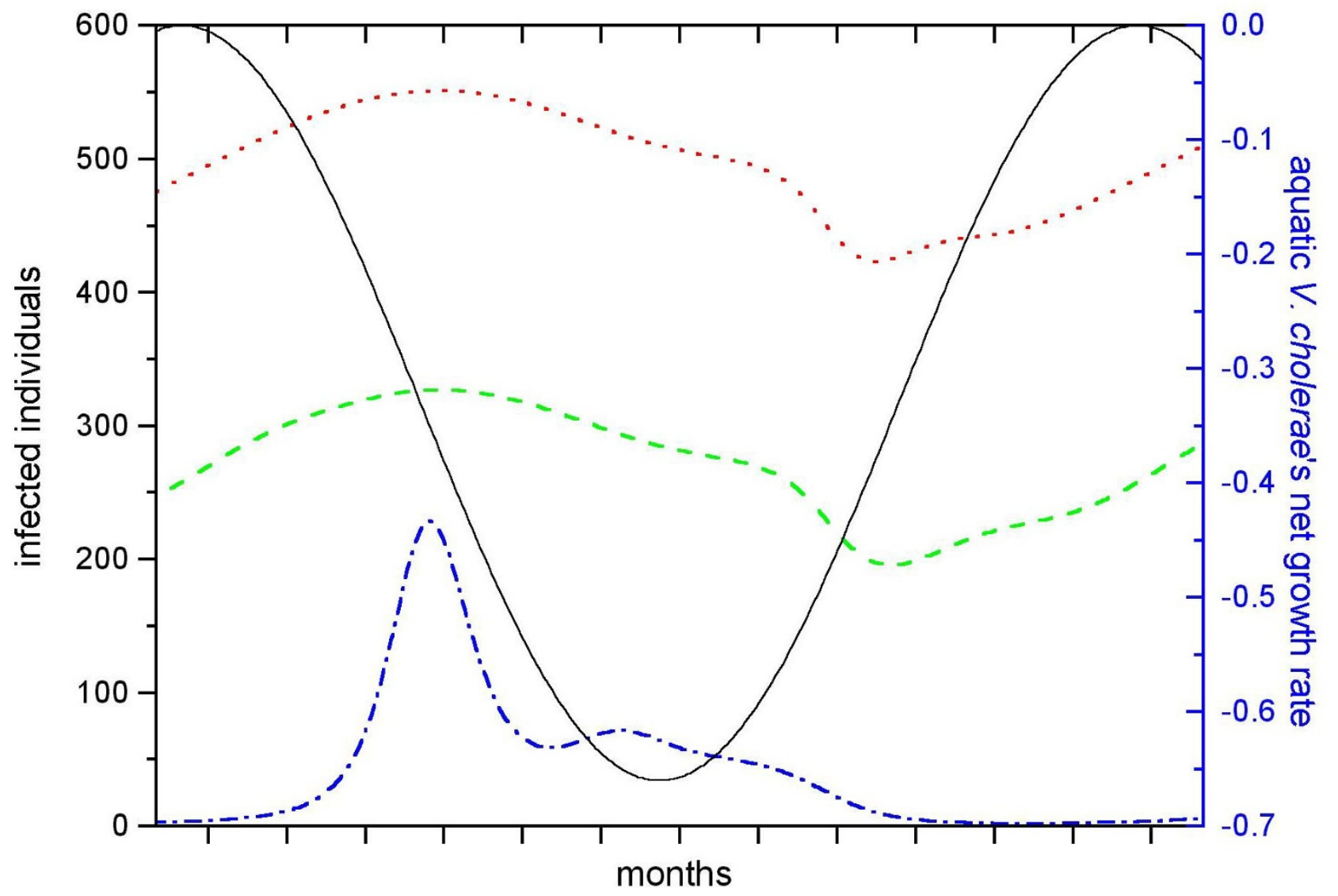

\section{Figure 8}

Dynamics of infection in a population with 1,$000 ; 5,500$ and 100,000 individuals in contact with a environmental reservoir of toxigenic $V$. cholerae. In this reservoir, growth rate of $V$. cholerae oscillates due to variations in water temperature, for example. Line symbols are the same of figure 6 . As in figures 6 and 7 , seasonal growth of $V$. cholerae in the environment also triggers seasonal outbreaks of cholera.

The probability of caching a $V$. cholerae infection is dosedependent. It is likely a minimal reservoir should be required for the occurrence of cholera cases in the population.

Endemic cholera, however, can be maintained even in the absence of a permanent reservoir $(m b>n b)$. This is a less obvious result. Endemism is maintained if bacteria net loss rate $(m b-n b)$ is sufficiently low to maintain an aquatic population of $V$. cholerae until the susceptible pool crosses the threshold $S_{\mathrm{C}}$ again. However, the best the sanitation condition (low $a$ and $e$ ), the longer-lived the reservoir must be. On the other hand, the worse the sanitation (high $a$ and $e$ ), the shorter can be the bacterial residence time in the water. In the limit, we may say that endemism in sanitized communities requires a permanent reservoir while endemism in poor communities requires just transient reservoirs (and a sufficiently high turnover of susceptibles).
This result suggests that cholera endemism in the US Golf Coast, for example, may be due to a permanent reservoir of $V$. cholerae. In some African regions, on the other hand, poor conditions may maintain endemism even in the absence of an environmental reservoir [4] [24].

In some cholera endemic regions, outbreaks are periodic with one or two peaks per year. Seasonal floods and droughts cause periodic variations in contact and contamination rates. If such variations are strong enough to cause large variations of $R_{0}$, we will note the occurrence of periodic outbreaks. Cholera outbreaks will be triggered when contact and/or contamination rates bring the $\mathrm{R}_{\mathrm{O}}$ above the unity threshold. This effect of contact rate variations on cholera dynamics was clearly observed in the Brazilian Amazon Region, during the 1990's. This region is characterized by seasonal flooding of the Amazon and Negro Rivers. Annual variations of water level 


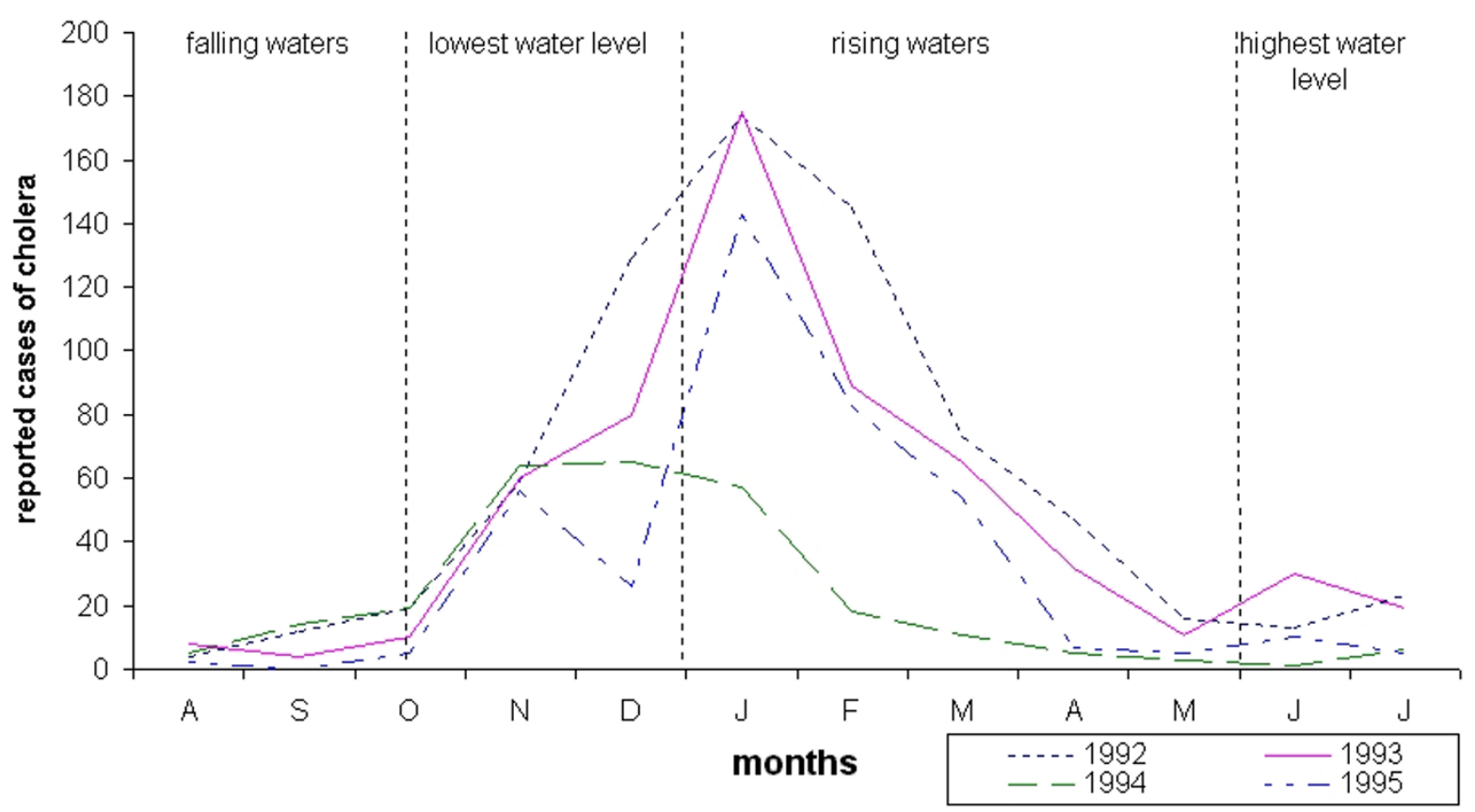

\section{Figure 9}

Periodicity of reported cases of cholera in the Brazilian Central Amazon region. This region is characterized by seasonal flooding of the Negro and Amazon Rivers, driven mainly by snow melt in the Andean headwaters of the Amazon River.

are quite high (ca. 8- 11 meters in Manaus) with slow rising and falling waters mainly driven by the slow snow melt in the Amazon River Andean headwaters [25]. The current cholera pandemics arrived in this region in 1991, caused outbreaks of marked periodicity (Figure 9). Cholera outbreaks started during the dry season where water is at the lowest level. During this period, potable water is often scarce and risk of contamination is high (high $e$; high $a$ ). Cholera peak coincides with the beginning of the rising waters. From this point on, cholera cases decrease until reaching its lowest levels during the high water period. This pattern is consistent with periodic variation of either $e$ or $a$ (Figures 6 and 7).

\section{Conclusion}

This work is an effort towards the formulation of a cholera theory that incorporates a $V$. cholerae environmental reservoir. The model proposed is very simple and does not include many features of this complex system. Nonetheless, this study brings some new insights into cholera epidemiology. Most studies on cholera epidemiology concentrate on either social or environmental factors. This work, however, shows that the reproduction rate of cholera is a function of social and environmental factors. It is necessary to determine the relative weights of each one of these components in order to develop appropriate control strategies.

Further developments on cholera modeling require a better understanding of $V$. cholerae ecology and epidemiology. We need estimates of the prevalence of $V$. cholerae infection in endemic populations. We also need better estimates of the required infection dose as well as a better description of the relationship between dose and virulence. 


\section{Appendix}

\section{Stability analysis.}

[http://www.biomedcentral.com/content/supplementary/14712334-1-1-s1.doc]

\section{Acknowledgments}

I would like to thank Dr. Bradley Sack and Dr. Russek-Cohen for providing critiques of this manuscript. Support was provided by FAPERJ, FINEP and PRONEX.

\section{References}

I. Epstein PR: Algal blooms in the spread and persistence of cholera. Biosystems | 1993, 31:209-221

2. Islam MS, Miah MA, Hasan MK, Sack RB, Albert MJ: Detection of non-culturable Vibrio cholerae $O I$ associated with a cyanobacterium from an aquatic environment in Bangladesh. Transactions of the Royal Society of Tropical Medicine and Hygiene 1994, 88:298-299

3. Faruque SM, Albert MJ, Mekalanos Jj: Epidemiology, genetics and ecology of toxigenic Vibrio cholerae. Microbiology and Molecular Biology Reviews 1998, 62:1 30 I-1314

4. Morillon M, De Pina J], Husser JA, Baundet JM, Bertherat E, Martet G: Djibouti, histoire de deux épidémies de choléra: 1993-1994. Bull Soc Path Ex 1998, 9 1:407-4II

5. Islam MS, Drasar B, Bradley SR: Probable role of blue-green algae in maintaining endemicity and seasonality of cholera in Bangladesh: a hypothesis. J Diarrhoeal Dis Res 1994, I 2:245-256

6. Colwell RR, Huq A: Environmental reservoir of Vibrio cholerae, the causative agent of cholera. Annals of the New York Academy Society 1994, 740:44-54

7. Anderson RM, May RM: Infectious diseases of humans. Oxford: Oxford University Press; 1991,

8. Capasso V, Paveri-Fontana SL: A mathematical model for the 1973 cholera epidemic in the european mediterranean region. Rev Epidém et Santé Pub 1979, 27:12 |-1 32

9. Sengupta TK, Nandy RK, Mukhopadyay S, Hall RH, Sathyamoorthy V, Ghose AC: Characterization of a 20-k Da pilus protein expressed by a diarrheogenic strain of non-OI/non-O139 Vibrio cholerae. FEMS Microbiol. Letters 1998, 160:183-189

10. Sack DA, Cadoz M: Cholera vaccines In Plotkin AS, Orenstein WA. Vaccines. Philadelphia: WB Saunders Co; 1999.

II. Sack FH, Pierce NF, Hennessey KN: Gastric acid in cholera and non-cholera diarrhea. Bulletin of the World Heath Organization 1972, 47:3I-36

12. Clemens JD, Sack DA, Harris JR: ABO blood groups and cholera: new observations on specificity of risk and modification of vaccine efficacy. The Journal of Infectious Diseases 1989, 159:770-773

13. Glass RI, Holmgren J, Haley CE: Predisposition for cholera of individuals with $O$ blood group. Possible evolutionary significance. Am J Epidemiol 1985, I 2 1:79 I-796

14. Hornick RB, Music SI, Wenzel RP, Cash RA, Libonati JP, Snyder MJ, et al: The Broad Street pump revisited: response of volunteers to ingested cholera vibrios. Bulletin of the New York Academy of Medicine 1971, 47:||8I-||19|

15. Feachem R, Bradley D, Garelick H, Mara DD: Vibrio cholerae and cholera. In Sanitation and disease. Health aspects of excreta and wastewater menagement. John Wiley \& Sons; 1983, 297-325

16. Islam MS, Drasar B, Bradley DJ: Survival of toxigenic Vibrio cholerae 01 on a duckweed, Lemna minor. Transactions of the Royal Society of Tropical Medicine and Hygiene 1990, 84:422-424

17. Lobitz B, Beck L, Huq B, Wood B, Fuchs G, Faruque SM, et al: Climate and infectious diseases: use of remote sensing for detection of Vibrio cholerae by indirect measurement. Proceedings of the National Academic Society USA 2000, 97: I438-I 443

18. Lawoyin TO, Ogunbodede NA, Olumide EAA, Onadeko MO: Outbreak of cholera in Ibadan, Nigeria. European Journal of Epidemiology 1999, 15:367-370
19. WHO: Cholera in 1998. Weekly Epidemiological Recort 1999, 74:257-263

20. Franco AA, Fix AD, Prada A, Paredes E, Palomino JC, Wright AC, Johnson JÁ, McCarter R, Guerra H, Morris JG Jr: Cholera in Lima, Peru, correlates with prior isolation of Vibrio cholerae from the environment. Am J Epidemiol 1997, 148:1067-1075

21. Colwell R: Global climate and infectious disease: the cholera paradigm. Science 1996, 274:2025-

22. Levin BR, Tauxe R: Cholera: nice bacteria and bad viruses. Current Biology 1996, 6:1389-1391

23. Taylor RK: Virus on virus infects bacterium. Nature 1999, 399:3। $2-313$

24. Utsalo SJ, Eko FO, Umoh F, Asindi AA: Faecal excretion of Vibrio cholerae during convalescence of cholera patients in Calabar, Nigeria. European Journal of Epidemiology 1999, I 5:379-38।

25. Walker I: Amazonian streams and small rivers. In Limnology in Brazil. Edited by Tundisi JG, Bicudo CEM, Tundisi TM. Rio de Janeiro: ABCI SBL; 1995, 167-194

26. Nisbet RM, Gurney WSC: Modelling fluctuating populations. New York: John Wiley \& Sons; 1982,

\section{Pre-publication history}

The pre-publication history for this paper can be accessed here:

http://www.biomedcentral.com/content/backmatter/ 1471-2334-1-1-b1.pdf

Publish with BioMedcentral and every scientist can read your work free of charge

"BioMedcentral will be the most significant development for disseminating the results of biomedical research in our lifetime." Paul Nurse, Director-General, Imperial Cancer Research Fund

Publish with BMc and your research papers will be:

- available free of charge to the entire biomedical community

- peer reviewed and published immediately upon acceptance

- cited in PubMed and archived on PubMed Central

- yours - you keep the copyright

Submit your manuscript here:

http://www.biomedcentral.com/manuscript/ 